\title{
Usefulness of abdominal ultrasound and magnetic resonance cholangiopancreatography (MRCP) in the diagnosis of hepatobiliary disorders: A comparative study
}

\author{
Naglaa M Elsayed ${ }^{1,2}$, Hanan S Binyahib ${ }^{1}$, Leen J Albalbeesi ${ }^{1}$ \\ 1. Department of Diagnostic Radiology, Faculty of Applied Medical Sciences, KAU, KSA. 2. Department of Diagnostic \\ Radiology, Faculty of medicine, Cairo University, Egypt. \\ Correspondence: Naglaa M Elsayed. Address: Department of Diagnostic Radiology, Faculty of Applied Medical Sciences, \\ KAU, KSA. Email: naglaamostafaelsayed@yahoo.com
}

Received: March 28, 2016

DOI : $10.5430 /$ ijdi.v3n2p26

Online Published: May 5, 2016

URL: http://dx.doi.org/10.5430/ijdi.v3n2p26

\section{Abstract}

Objective: The aim of this work was to compare the usefulness and sensitivity of ultrasonography (US) and magnetic resonance cholangiopancreatography (MRCP) in the diagnosis of different hepatobiliary disorders.

Methods: The study included 65 patients with various hepatobiliary disorders such as cholelithiasis, post cholecystectomy complications, neoplastic, inflammatory and developmental conditions of the pancreatico-biliary system. All patients underwent an initial abdominal US followed by MRCP, allowing a direct comparison of the results obtained by these two modalities. Correlation was also made with available clinical records, other imaging modalities, intraoperative findings and histopathology results.

Results: While both modalities proved equally sensitive in revealing developmental and inflammatory diseases of the pancreatico-biliary system (values between 90\%-100\%), MRCP showed a higher sensitivity for detecting pancreatico-biliary tumors $(100 \%$ vs. $14.2 \% ; p<.05)$ and cholelithiasis $(96.2 \%$ vs. $74.0 \% ; p<.05)$. MRCP also demonstrated a slightly higher sensitivity in revealing post cholecystectomy complications ( $92.8 \% v s .78 .5 \%)$, even though the difference did not reach statistical significance $(p=.317)$. A variety of other findings and anatomical variants of the biliary and pancreatic ducts were revealed only by MRCP.

Conclusions: MRCP showed an overall higher sensitivity for revealing hepatobiliary disorders compared to abdominal US. The modality is especially useful when the findings revealed by abdominal US are inconclusive or when clinical suspicion persists despite negative US results.

\section{Keywords}

Magnetic resonance cholangiopancreatography, Abdominal ultrasound, Hepatobiliary disorders

\section{Introduction}

Diseases of the biliary system are common in medical practice. Due to this fact, diagnostic studies of the biliary system should be performed to rule them out. US is used for decades as a primary modality of investigation. It is an inexpensive, 
accurate, accessible, safe, dynamic and noninvasive imaging modality, which has a fast acquisition time with no contraindications. Indications for the US of the gall bladder (GB) and biliary system include patients with jaundice, abnormal liver function tests, suspected cholangitis or suspected gallstones. It has been proven that ultrasonography (US) has a high diagnostic accuracy (> 90\%) for detecting a variety of conditions such as biliary strictures, cholelithiasis, cholangiocarcinoma and periampullary cancers. US is also a useful modality for evaluating patients with suspected acute and chronic cholecystitis, biliary dilatation, as well as for defining the level of biliary obstruction ${ }^{[1]}$. At the same time the modality is highly operator dependent and may provide limited information in obese individuals, patients with surgical dressings or gaseous distention of the abdomen.

MRCP is another non-invasive technique used for imaging the biliary system. It is commonly employed when relevant diagnostic questions could not be answered by abdominal US or before proceeding to more invasive techniques like ERCP. Common indications include suspected diseases of the liver, gall bladder, biliary and pancreatic duct system of various etiologies as well as evaluation of postoperative results after hepatobiliary surgical procedures. MRCP is commonly performed on a 1.5T MRI scanner using abdominal phased array body coils. Heavily T2 weighted images are obtained using both breath-hold and non-breath-hold sequences. Special MRI protocols such as rapid acquisition and relaxation enhancement (RARE), fast-recovery fast spin-echo coronal oblique 3D respiratory triggered (FRFSE) and halfourier acquisition single shot turbo spin echo (HASTE) are also frequently used for MRCP ${ }^{[2]}$. Even though MRCP is non-invasive and has the advantage of adding a 3D imaging and fast multiple planes, it represents a purely diagnostic technique without any options to correct or treat the identified problems during the procedure ${ }^{[3]}$.

\section{Materials and methods}

\subsection{Subjects}

This retrospective study included 65 patients (41 females and 24 males), ranging in age from 1 to 88 years old, with various hepatobiliary disorders such as cholelithiasis, neoplastic, inflammatory and developmental conditions of the pancreatico-biliary system. Among these, 14 patients presented with post-cholecystectomy complications, most interventions being performed laparoscopically. All patients were referred for initial abdominal US followed by MRCP, allowing a direct comparison of the results obtained by these two modalities. Correlation was also made with available clinical records, other imaging modalities, intraoperative findings and histopathology results. The study was done between September 2014 and May 2015 at the diagnostic radiology department, KAUH after obtaining an ethical approval from the faculty of applied medical sciences ethical committee. All patients provided written consents before MRCP examination.

\subsection{US technique}

US of the biliary system was performed using a Philips 22 machine and a curved array transducer with C5-10 MHz frequency in sagittal and axial plans. Imaging the intrahepatic biliary system was performed starting from the left lobe of liver then proceeding to the right lobe. Then, the common bile duct and gallbladder were scanned. Doppler application was used to identify the CBD from the portal vein and hepatic artery. Then the pancreas was scanned for identifying the pancreatic duct. Patients were asked to fast 6-8 hours before US examination to facilitate distention of the gallbladder and to minimize image degradation by gaseous distention of the bowel.

\subsection{MRCP technique}

MRCP was performed on a $1.5 \mathrm{~T}$ Siemens MRI scanner using a phased-array coil.

(1). Three plan localizing images were obtained and used to plan MRCP sequence.

(2). AX-T2-FS-NAV

- $\quad$ Field of view $=330 \mathrm{~mm}$

Published by Sciedu Press 
- $\quad$ Slice thickness $=5 \mathrm{~mm}$

(3). AX-T2- BH

- $\quad$ Field of view $=330 \mathrm{~mm}$

- $\quad$ Slice thickness $=5 \mathrm{~mm}$

(4). AX-T2_HASTE290_TE

(5). AX T1 IN-OUT PHASE_ABD

- $\quad$ Field of view $=330 \mathrm{~mm}$

- $\quad$ Slice thickness $=5 \mathrm{~mm}$

(6). COR HASTE THICK

- $\quad$ Field of view $=250 \mathrm{~mm}$

- $\quad$ Slice thickness $=50 \mathrm{~mm}$

(7). T1-fL2D-TRA-P2-MBH

- $\quad$ Field of view $=330 \mathrm{~mm}$

- $\quad$ Slice thickness $=4 \mathrm{~mm}$

(8). COR 3D-MRCP

- $\quad$ Field of view $=300 \mathrm{~mm}$

- Slice thickness $=1.30 \mathrm{~mm}$

The coverage area extended from the nipple line to the iliac crest. To promote gallbladder filling, patients were asked to fast for 4 to 6 hours. All sequences were acquired during a single breath-hold. The whole examination was regularly completed within 20 minutes with the patient in the supine position. No anesthesia or contrast agents were used.

\subsection{I mage interpretation}

US was done by expert sonographers and reviewed by radiologists specialized in abdominal US, while MRCP examination was interpreted by expert radiologists specialized in body MRI. The following parameters were studied: the gall bladder distension, wall thickness, pericholecystic fluid collection and the presence of stones or masses. The intra and extrahepatic biliary radicles were evaluated regarding their diameter, the presence of stones, stents or anatomical variations. During US examinations, Murphy's sign was applied in cases of clinically suspected acute cholecystitis. Associated findings such as pancreatic or hepatic masses, liver cirrhosis or abdominal collection were also recorded.

\subsection{Follow up and clinical outcomes}

Follow up of patients was done till reaching a final definitive diagnosis. Some patients required further investigation using a more invasive diagnostic procedure like endoscopic retrograde cholangiopancreatography (ERCP) (15 patients), percutaneous transhepatic pancreatography (PTC) (1 patient), or computed tomography (CT) (3 patients). Other patients were subjected to surgical intervention through laparoscopic cholecystectomy (19 patients), laparotomy (4 patients) or partial hepatectomy (2 patients). One patient with a malfunctioning biliary stent underwent repeated stenting for biliary obstruction. In 2 patients, biopsy and histopathology results were obtained to reach a final diagnosis. Follow up by US or MRCP was asked for 7 patients. The remaining patients required only medical treatment before they were discharged from the hospital (11 patients). All patients provided written consents before invasive procedures and operative intervention.

\subsection{Statistical analysis}

The results obtained by the two imaging modalities were compared using McNemar's test. The required statistical calculations were performed using a specially designed DTCompair package ${ }^{[4]}$. A $p$-value $<.05$ was considered significant. The specificity values have not been calculated in this study because there were no true negative cases among the admitted patients. 


\section{Results}

About $2 / 3$ of our patients (63.1\%) were females and about $1 / 3$ (36.9\%) were males, the data suggesting a relatively higher frequency of biliary pathology in females. The types of detected disorders are provided in Figure 1, while the number of encountered cases for different entities is shown in Table 1. Cholelithiasis represented the most frequent pathology (41\%), followed by a variety of post-cholecystectomy complications (19\%), inflammatory disorders (15\%) and neoplastic conditions (11\%). Most conditions were detected by both US and MRCP, even though the sensitivity of MRCP was significantly higher compared to that of US (93.8\% vs. $72.3 \%, p=.001)$.

Figure 1. General distribution of biliary disorders among the study population

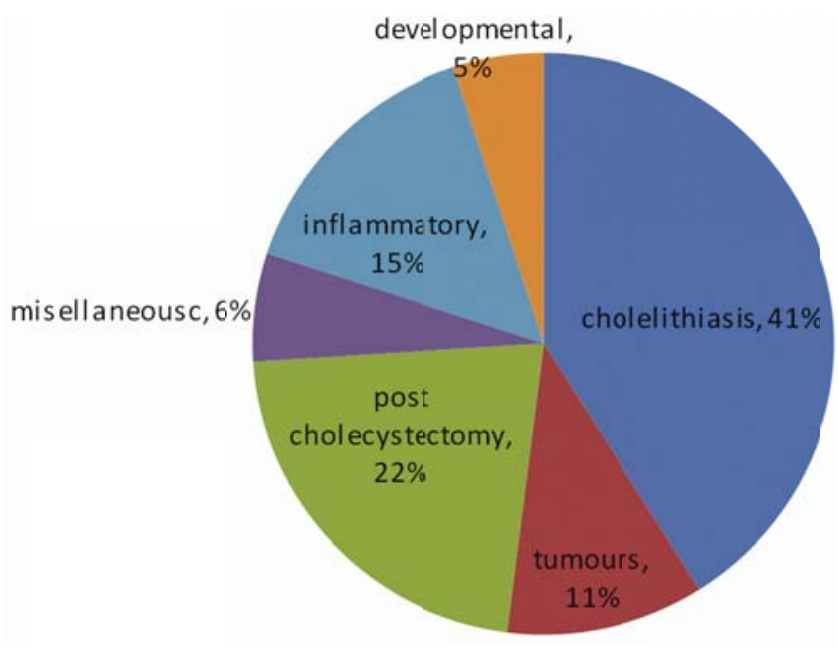

\subsection{Cholelithiasis}

From a total of 27 patients with cholelithiasis (see Table 1), 20 were diagnosed correctly by both US and MRCP. In another 6 patients, gallstones could be detected only by MRCP ( 5 of these showing negative findings on US and 1 case being misdiagnosed as a calcified gallbladder polyp). One case of cholelithiasis was missed by both modalities, being diagnosed as acalculous cholecystitis; however, the intra-operative findings subsequently reported biliary stones. Thus, the overall sensitivity for detecting cholelithiasis was $74.0 \%$ for US and $96.2 \%$ for MRCP, the difference being statistically significant $(p=.014)$. Representative examples of imaging findings obtained by both modalities are provided in Figure 2.
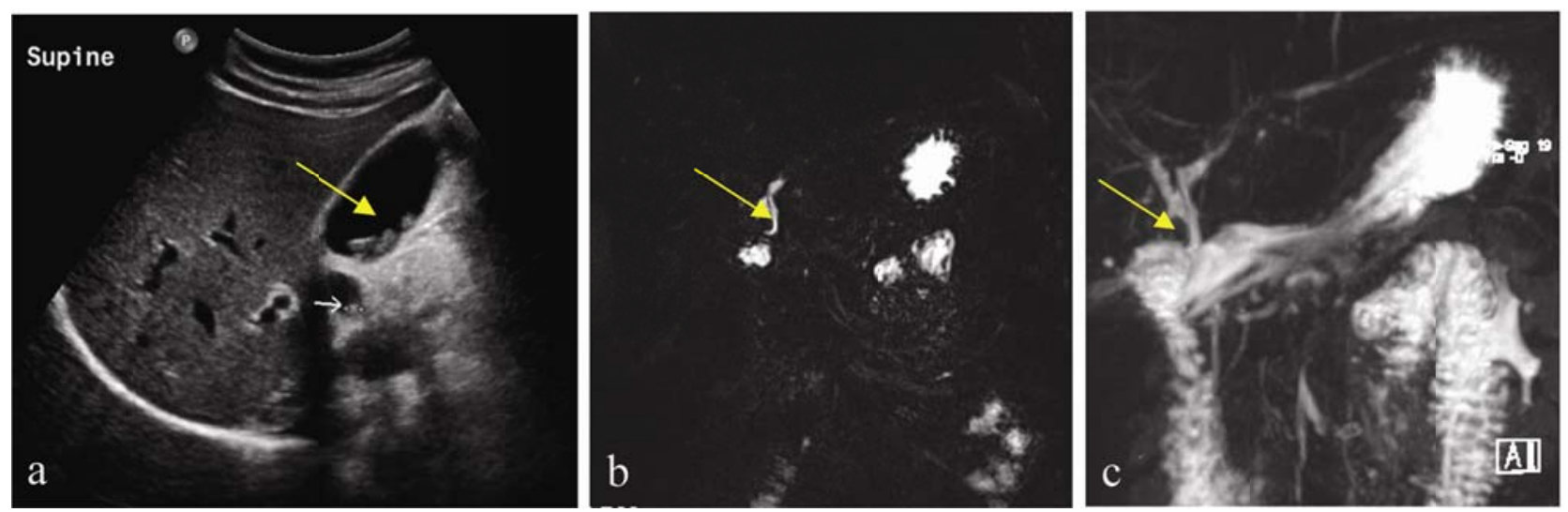

Figure 2. Abdominal US in supine position showing multiple biliary stones in the gallbladder (panel a, yellow arrow), including a small gallstone in the gallbladder neck (panel a, small white arrow). MRCP images displaying a gallstone in the common hepatic duct, seen as a filling defect (panels b-c, arrows) 


\subsection{Inflammatory diseases of the pancreatico-biliary system}

From a total of 10 patients with cholecystitis or pancreatitis (see Table 1), 8 were diagnosed correctly by both US and MRCP. One case of associated chronic cholecystitis in a patient with gallstones was described only by US (MRCP revealing only the gallstones) and one case of chronic pancreatitis was diagnosed only by MRCP (no significant changes in pancreatic parenchyma described on US). Hence, US and MRCP showed similar sensitivities (90\%) for detecting inflammatory diseases of the pancreatico-biliary system in this study.

Table 1. Distribution of pathological findings among the study population

\begin{tabular}{|c|c|}
\hline Diagnosis & Number of patients \\
\hline Cholelithiasis & 27 \\
\hline GB & 21 \\
\hline CBD & 2 \\
\hline CHD & 1 \\
\hline GB sludge & 2 \\
\hline IHBR & 1 \\
\hline Developmental anomalies & 3 \\
\hline Caroli disease & 1 \\
\hline Choledocal cyst & 2 \\
\hline Inflammatory disorders & 10 \\
\hline Acute calcular cholecystitis & 4 \\
\hline Acute pancreatitis & 1 \\
\hline Chronic calcular cholecystitis & 3 \\
\hline Chronic pancreatitis & 2 \\
\hline Miscellaneous & 4 \\
\hline Chronic cholangitis & 1 \\
\hline Liver abscess & 1 \\
\hline Malfunctioning stent & 1 \\
\hline pneumobilia & 1 \\
\hline Post cholecystectomy findings & 14 \\
\hline Abdominal collection & 3 \\
\hline Anastomotic stricture & 1 \\
\hline Biliary dilatation & 5 \\
\hline Biliary stricture & 1 \\
\hline Non biliary cause of jaundice & 2 \\
\hline Residual cholelithiasis & 2 \\
\hline Tumors & 7 \\
\hline Cholangiocarcinoma & 4 \\
\hline Pancreatic head tumor & 2 \\
\hline Periampullary adenocarcinoma & 1 \\
\hline
\end{tabular}

\subsection{Pancreatico-biliary tumors}

Pancreatico-biliary tumors were detected in 7 patients (see Table 1), the diagnosis being confirmed histologically in all cases (tissue sampling obtained by ERCP, CT guided biopsy and/or resected surgical specimens). All tumors were correctly diagnosed by MRCP. A certain diagnosis by US, however, was made only in one patient with a pancreatic head tumor. In the remaining 6 patients, the US findings were either equivocal or negative for neoplastic lesions. Thus, the sensitivity of MRCP for detecting pancreatico-biliary tumors in our study was $100 \%$, whilst the sensitivity of US was only $14.2 \%(p=.014)$. An example of imaging findings obtained by both modalities in a patient with cholagniocarcinoma is shown in Figure 3. 

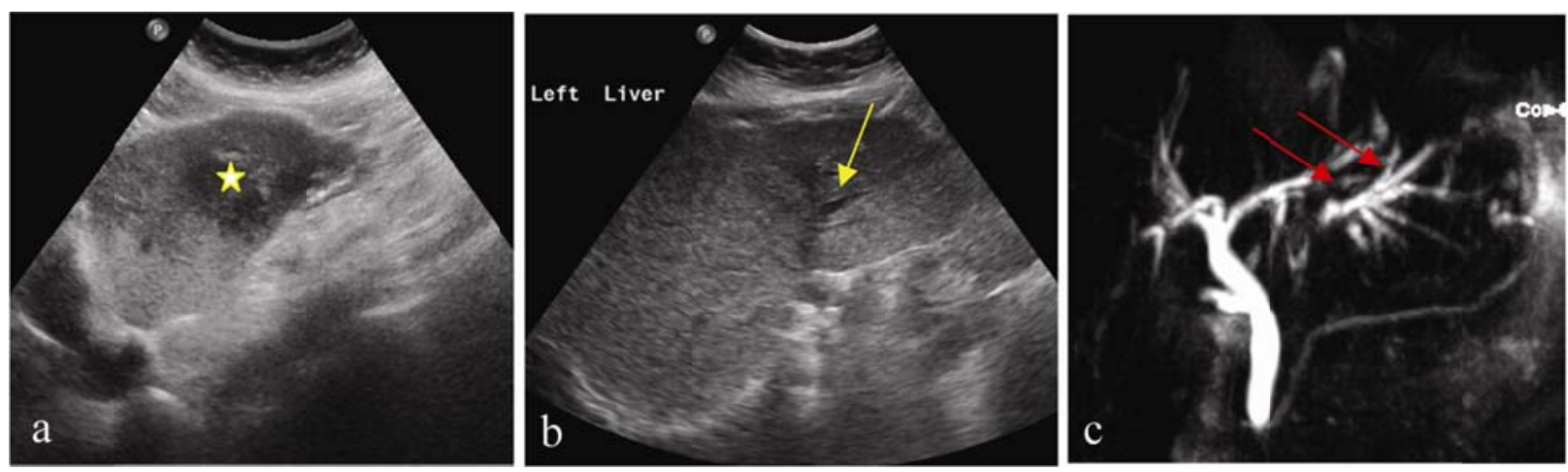

Figure 3. Abdominal US showing a non-specific hypoechoic mass in the left lobe of the liver (panel a, star) and adjacent biliary dilatation (panel b, yellow arrow). MRCP of the same patient reveals prominently dilated intrahepatic biliary system in the left hepatic lobe (panel c, red arrows). Cholagniocarcinoma was subsequently confirmed by histopathology

\subsection{Post cholecystectomy complications}

A total of 14 patients presented with various post-cholecystectomy complications (see Table 1). In 10 patients the complications were diagnosed correctly by both US and MRCP. In another 3 patients, the cause of their symptoms could be revealed only by MRCP (residual stones in the intrahepatic bile ducts, a stricture of the common hepatic duct and a stricture of the left hepatico-jeujenal anastomosis, which is also displayed in Figure 4). On the contrary, a case of biliary cirrhosis responsible for persistent postoperative jaundice was revealed only on the US exam. Even though the sensitivity of MRCP for revealing post-cholecystectomy complications appeared higher (92.8\%) compared to the sensitivity of US (78.5\%), the difference did not reach statistical significance in our study $(p=.317)$.

Figure 4. MRCP showing a stricture of the left hepatico-jeujenal anastomosis (red arrows) and dilated left intrahepatic biliary ducts. The yellow arrow points to the jejunum
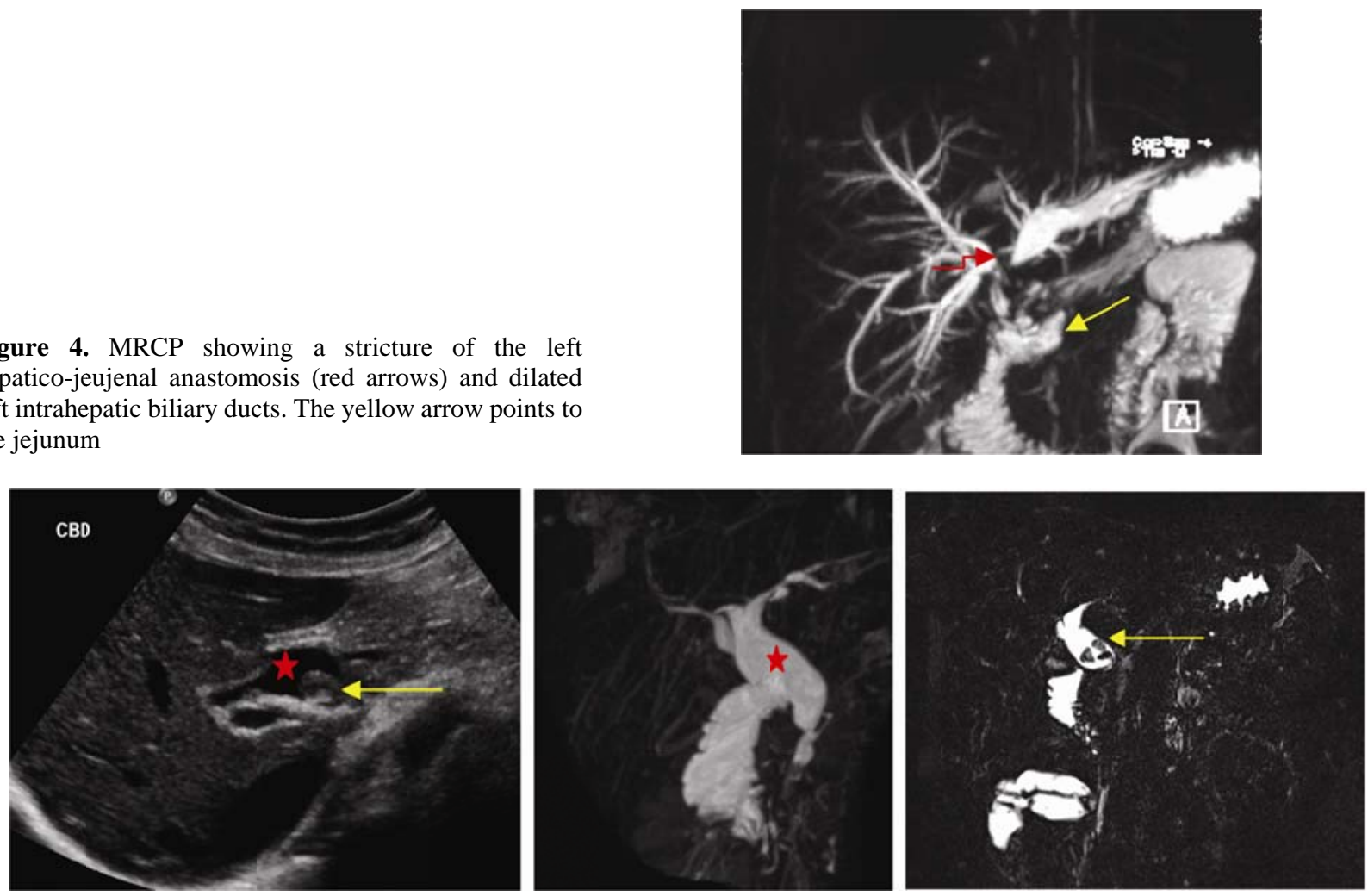

Figure 5. Liver US (panel a) showing a fusiform dilatation of the common bile duct (star) that contains a distinct filling defect (arrow). MRCP performed in the same patient also demonstrates a fusiform dilatation of the common bile duct panel b, star) with multiple signal void structures (panel c, arrow). The patients had a choledochal cyst containing multiple stones 


\subsection{Developmental anomalies}

Developmental anomalies of the biliary system were detected in 3 patients: one patient included in this study had Caroli Disease and two patients had choledochal cysts type I (one of them being displayed in Figure 5). All 3 cases were correctly diagnosed by both US and MRCP, reflecting a sensitivity of $100 \%$ for both modalities. However, given the small number of cases included in this study, the results may need confirmation in larger cohorts.

\subsection{Miscellaneous cases}

Miscellaneous hepatobiliary pathology in this study was represented by such entities as pneumobilia (1 patient), chronic cholangitis (1 patient), a malfunctioning biliary stent and a hepatic abcess (see Table 1). Pneumobilia was diagnosed only by US, the chronic cholangitis was diagnosed only by MRCP, while the hepatic abscess and the malfunctioning biliary stent were revealed by both modalities. The overall sensitivity for detecting hepatobiliary pathology was $75 \%$ for both modalities. However, given the small number of cases included in this study, these results also need confirmation in larger cohorts. Moreover, a variety of other findings and details were revealed by MRCP only (see Figure 6), underlying the usefulness of this modality and the advantages it may have in certain categories of patients. A representative case is shown in Figure 7.

Figure 6. Relevant findings detected by MRCP only

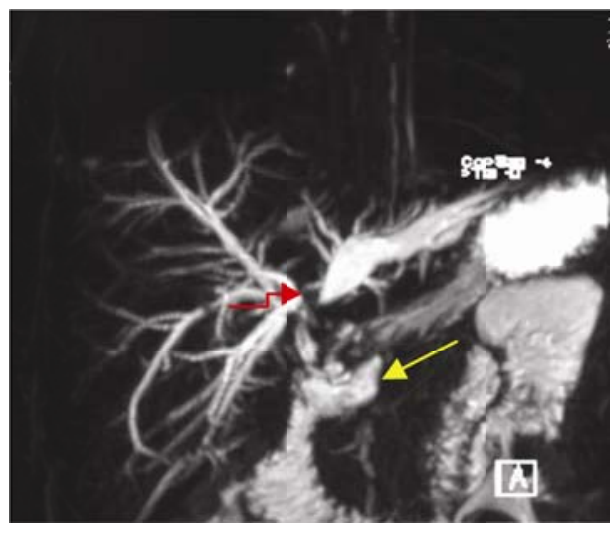

Figure 7. MRCP revealing an elongated vertical course of the pancreatic duct (yellow arrow), which subsequently joins the common bile duct (red arrow) before their insertion into the third part of the duodenum (white arrow)

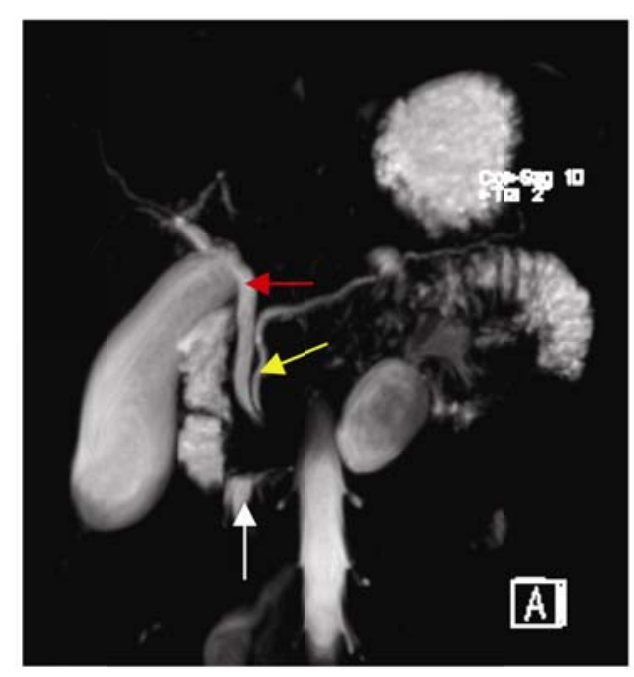

\section{Discussion}

In this study, MRCP showed an overall higher sensitivity for revealing hepatobiliary disorders compared to abdominal US. The study design also allowed comparing the usefulness of the two imaging modalities in patients with specific hepatobiliary entities such as cholelithiasis, post cholecystectomy complications, neoplastic, inflammatory and developmental conditions of the pancreatico-biliary system. 


\subsection{Cholelithiasis}

Cholelithiasis accounts for most cases of obstruction of bile ducts. Direct cholangiography is generally still considered to be the ideal method for diagnosing CBD calculi ${ }^{[5]}$. On US, calculi typically appear as echogenic structures within the GB with posterior acoustic shadows. On MRCP calculi are identified as signal voids within the high signal intensity fluid in the bile ducts. The differential diagnosis of these signal voids includes air bubbles, blood clots, sludge ball, flow voids and susceptibility artifacts from surgical clips ${ }^{[6]}$. In our study, from a total of 27 patients with cholelithiasis, 26 were diagnosed correctly by MRCP and only 20 by US, translating into a sensitivity of $96.2 \%$ for MRCP and $74.0 \%$ for US. Even though the abdominal US has a relatively high sensitivity for detecting biliary stones, the even higher values obtained for MRCP in the current study are directly related to the ability of MRCP to detect stones of the biliary radicles that were missed by US. In the literature, the reported US sensitivity to detect biliary stones is variable, ranging from $20 \%$ to $80 \%{ }^{[7]}$. Nevertheless, US remains the imaging method of choice for diagnosis of gall bladder pathology. Although MRCP demonstrated a higher sensitivity in the current study, additional imaging studies may be also necessary for reaching a final diagnosis in selected patients.

\subsection{I nflammation of the pancreatico-biliary system}

Imaging findings of acute cholecystitis include gallbladder over-distension with intra-luminal sludge or stones, mural thickening and edema, pericholecystic fluid, and positive Murphy's sign. Chronic cholecystitis, however, may be associated with less evident changes and evaluation of gallbladder contractility may be required in some cases. US is the primary imaging modality in suspected cholecystitis, with a reported sensitivity between $37.5 \%-91 \%$ and specificity between $60 \%-100 \%$. MRCP is reported to have a higher accuracy in acute cholecystitis, with sensitivity between $88 \%-95 \%$ and specificity between $69 \%-89 \%{ }^{[8]}$. Acute pancreatitis is another clinical condition that can be triggered by gallstones. The aim of imaging is not only to detect the pancreatitis, but also to localize the biliary stones responsible for the attacks. The sensitivity and specificity of abdominal ultrasound in diagnosing acute pancreatitis is around $73.6 \%$ and $97.7 \%$, respectively ${ }^{[9]}$. MRCP has recently been evolving as an important tool for the evaluation of chronic pancreatitis with a sensitivity of $92 \%$ and a specificity of $75 \%$ for the demonstration of early chronic pancreatitis ${ }^{[10]}$. In the current study, US and MRCP showed similar sensitivities (90\%) for detecting inflammatory diseases of the pancreatico-biliary system. However, due to a relatively small number of such patients $(n=10)$, the findings need confirmation in larger cohorts.

\subsection{Pancreatico-biliary tumors}

Cholangiocarcinoma is a malignant tumour arising from cholangiocytes in the biliary tree. It tends to have a poor prognosis and high morbidity ${ }^{[11]}$. Risk factors for cholangiocarcinoma are choledochal cysts, caroli's disease, liver cirrhosis, primary sclerosing cholangitis and hepatitis B virus infection ${ }^{[12]}$. The sensitivity of MRCP for concurrent cholangiocarcinoma reaches up to $87 \%{ }^{[13]}$. In the current study we examined 7 patients with pancreatico-biliary tumors, including 4 cases of cholangiocarcinomas, the diagnosis being confirmed histologically in all patients. All tumors were correctly diagnosed by MRCP, the modality demonstrating $100 \%$ sensitivity. This was in contrast with the abdominal US exam, which demonstrated a sensitivity of only $14.2 \%$ (a certain diagnosis of a pancreatico-biliary tumor being made by US only in 1 patient). The results are in overall agreement with the findings reported by other authors. Thus, Tse et al. reported that MRCP is very useful in the diagnosis of cholangiocarcinoma, identifying the exact location, extent, and severity of the obstruction ${ }^{[14]}$. Miura et al. pointed out that the accuracy of conventional US for diagnosing pancreatic tumors is only $50 \%-70 \%{ }^{[15]}$. Delden et al. further emphasized that in the detection of pancreatic cancers, US has an overall sensitivity of $75 \%$ and a specificity of $75 \%$, while the sensitivity of US for detecting ampullary carcinoma is as low as $5 \%{ }^{[16]}$. In our study we had one case of periampullary carcinoma, which was correctly diagnosed only by MRCP.

\subsection{Post-cholecystectomy complications}

Post-cholecystectomy syndrome (PCS) consists of a group of abdominal symptoms that recur and/or persist after cholecystectomy including abdominal pain, dyspepsia, vomiting, gastrointestinal disorders and jaundice, with or without 
fever and cholangitis. It may occur early or as late as months or years after cholecystectomy ${ }^{[17]}$. It includes a large number of disorders, both biliary and extra-biliary (gastrointestinal, extra intestinal or psychomotor) in origin that may be even unrelated to cholecystectomy. Moreover, in 5\% of patients who undergo laparoscopic cholecystectomy, the reason for chronic abdominal pain remains unknown ${ }^{[17]}$. The reported prevalence of post cholecystectomy complications ranges from very low to $47 \%{ }^{[17]}$. A relatively common finding after colecystectomy is biliary dilatation and a diameter of the CBD within $10 \mathrm{~mm}$ can be regarded as normal ${ }^{[18]}$. The reported sensitivity of MRCP for biliary strictures ranges from $78 \%$ to $100 \%{ }^{[19]}$. In another study, the reported sensitivity, specificity and diagnostic accuracy of MRCP were $100 \%, 88.23 \%$, and $94.87 \%$, respectively ${ }^{[6]}$. In our study, 14 (21\%) patients presented with post cholecystectomy complications. MRCP showed a relatively higher sensitivity for detecting these complications compared to abdominal US (92.8\% vs. 78.5\%), even though the difference did not reach statistical significance $(p=.317)$. Post-operative fluid collections were easily diagnosed by both US and MRCP, even though MRCP could also detect a biliary fistula responsible for the collection in one case.

\subsection{Developmental anomalies}

Choledochal cysts represent congenital cystic dilatations of the biliary tree. Patients usually present with abdominal pain, jaundice and an abdominal mass. The two most frequent complications of choledochal cysts are stone formation and malignancies ${ }^{[20]}$. In the current research, 2 cases of choledochal cysts were diagnosed by both MRCP and US. The findings are consistent with those reported in the literature, indicating an overall detection rate of choledochal cysts by MRCP between $96 \%-100 \%{ }^{[13,21]}$.

\subsection{Malfunction stent}

The commonest stent related complications are recurrent cholangitis and stent migrations, followed by pancreatitis and cholecystitis. Clinically it may present with recurrent signs of biliary obstruction and cholangitis ${ }^{[22]}$. Our study included one case of a malfunctioning biliary stent, which was correctly revealed by both imaging modalities. The patient, who had a pancreatic head tumor, presented with persistent obstructive jaundice after his biliary stent insertion. Both MRCP and US show marked biliary dilatation due to malfunctioning stent.

\subsection{Anatomical variation of the biliary system}

Apart from detecting hepatobiliary pathology, revealing anatomic variants of biliary and pancreatic duct system may be equally important, especially for patients considered for biliary surgery. MRCP can reveal a variety of anatomical variants such as low or medial cystic duct insertions, aberrant right hepatic ducts or a parallel course of cystic and hepatic ducts with an accuracy between $95 \%-98 \%{ }^{[6]}$. Such information can be useful for planning the surgical intervention as well as for avoiding intraoperative complications ${ }^{[6]}$. Given the information it can provide and the non-invasiveness of the technique, MRCP is being increasingly considered as an alternative to ERCP in suitable patients ${ }^{[23]}$. In our study, MRCP was able to reveal anatomical variants of cystic duct insertion in 7 patients and an anatomical variant of pancreatic duct insertion in 1 patient. None of these could be visualized on abdominal US.

In summary, while both modalities proved equally sensitive in revealing developmental and inflammatory diseases of the pancreatico-biliary system (values between 90\%-100\%), MRCP showed a higher sensitivity for detecting pancreatico-biliary tumors $(100 \%$ vs. $14.2 \%$; $p .05)$ and cholelithiasis $(96.2 \%$ vs. $74.0 \%$; $p<.05)$. MRCP also demonstrated a slightly higher sensitivity in revealing post cholecystectomy complications ( $92.8 \%$ vs. $78.5 \%)$, even though the difference did not reach statistical significance $(p=.317)$. A variety of other findings and anatomical variants of the biliary and pancreatic ducts were revealed only by MRCP.

\section{Conclusions}

MRCP showed an overall higher sensitivity for revealing hepatobiliary disorders compared to abdominal US. The modality is especially useful when the findings revealed by abdominal US are inconclusive or when clinical suspicion 
persists despite negative US results. Given its high sensitivity for revealing anatomical variants of hepatobiliary and pancreatic duct system, MRCP can be especially useful in patients considered for biliary duct surgery and may also serve as an alternative to ERCP in suitable patients.

\section{References}

[1] Singh A, Mann H, Thukral C, et al. Diagnostic Accuracy of MRCP as Compared to Ultrasound/CT in Patients with Obstructive Jaundice. J Clin Diagn Res. 2014; 8(3): 103-107. http://dx.doi.org/10.7860/jcdr/2014/8149.4120

[2] Mudgal P. Magnetic resonance cholangiopancreatography/Radiology Reference Article/Radiopaedia.org [Internet]. Radiopaedia.org. 2015 [cited 25 April 2015]. Available from: http://radiopaedia.org/articles/magnetic-resonance-cholangiopancreatography.

[3] Ihe-online.com. MRCP versus ERCP in the evaluation of biliary pathologies [Internet]. 2015 [cited 13 January 2015]. Available from: http://www.ihe-online.com/feature-articles/mrcp-versus-ercp-in-the-evaluation-of-biliary-pathologies/

[4] Christian Stock, Thomas Hielscher. DTComPair: comparison of binary diagnostic tests in a paired study design. R package version 1.0.3. 2014. Available from: http://CRAN.R-project.org/package=DTComPair

[5] Norero E, Norero B, Huete A, et al. Accuracy of magnetic resonance cholangiopancreatography for the diagnosis of common bile duct stones. Rev Med Chil. 2008; 136: 600-5. PMid:18769807

[6] Parashari UC, Khanduri S, Bhadury S, et al. Diagnostic role of magnetic resonance cholangiopancreatography in evaluation of obstructive biliopathies and correlating it with final diagnosis and clinical profile of patients. J Nat Sci Biol Med. 2015; 6(1): 131-138. PMid:25810650 http://dx.doi.org/10.4103/0976-9668.149110

[7] Ferrari FS, Fantozzi F, Tasciotti L, et al. US, MRCP, CCT and ERCP: A comparative study in 131 patients with suspected biliary obstruction. Med Sci Monit. 2005; 11(3): 8-18.

[8] Tonolini M, Ravelli A, Villa C, et al. Urgent MRI with MR cholangiopancreatography (MRCP) of acute cholecystitis and related complications: diagnostic role and spectrum of imaging findings. Emerg Radiol. 2012; 19(4): 341-8. PMid:22447440 http://dx.doi.org/10.1007/s10140-012-1038-z

[9] Prasad H, Rodrigues G, Shenoy R. Role of Ultrasonography in Non Traumatic Acute Abdomen. The Internet Journal of Radiology. 2006; 5: 2-7. PMid:17238039 http://dx.doi.org/10.1007/s00535-006-1919-6

[10] Czakó L. Diagnosis of early-stage chronic pancreatitis by secretin-enhanced magnetic resonance cholangiopancreatography. J Gastroenterol. 2007; 42(17): 113-7.

[11] Gaillard F. Cholangiocarcinoma/Radiology Reference Article/Radiopaedia.org [Internet]. Radiopaedia.org. 2015 [cited 22 May 2015]. Available from: http://radiopaedia.org/articles/cholangiocarcinoma

[12] Bragazzi MC, Cardinale V, Carpino G, et al. Cholangiocarcinoma: Epidemiology and risk factors. Transl Gastrointest Cancer. 2012; 1: 21-32.

[13] Park DH, Lee SK, Choi JS, et al. Can MRCP replace the Diagnostic Role of ERCP for Patients with Choledochal Cyst? Gastrointst Endosc. 2005; 6(3): 360-6. PMid:16111952 http://dx.doi.org/10.1016/j.gie.2005.04.026

[14] Tse F, Barkun J, Romanuolo J, et al. Nonoperative Imaging Technique in Suspected Biliary Tract Obstruction. HPB (Oxford). 2006; 8(6): 409-425. PMid:18333096 http://dx.doi.org/10.1080/13651820600746867

[15] Miura F, Takada T, Amano H, et al. Diagnosis of pancreatic cancer. HPB (Oxford). 2006; 8(5): 337-342. PMid:18333085 http://dx.doi.org/10.1080/13651820500540949

[16] Radiologyassistant.nl. (2006). The Radiology Assistant: Pancreas-Carcinoma. [online]. Available from: http://www.radiologyassistant.nl/en/p43848b63def9d/pancreas-carcinoma.html [Accessed 26 May 2015].

[17] Bilgin M, Toprak H, Burgazli M, et al. Diagnostic Value of Dynamic Contrast Enhancement Mgnetic Resonance Imaging in the Evaluation of the Biliary Obstruction. The Scientific Journal. 2012: 9.

[18] Park SM, Kim WS, Bae IH, et al. Common bile duct dilatation after cholecystectomy: a one-year prospective study J Korean Surg Soc. 2012; 83(2): 97-101. PMid:22880184 http://dx.doi.org/10.4174/jkss.2012.83.2.97

[19] Textor HJ, Flacke S, Pauleit D, et al. Three-dimensional magnetic resonance cholangiopancreatography with respiratory triggering in the diagnosis of primary sclerosing cholangitis: Comparison with endoscopic retrograde cholangiography. Endoscopy. 2002; 34: 984-90. PMid:12471543 http://dx.doi.org/10.1055/s-2002-35830

[20] Gaillard F. (2015). Choledochal cyst/Radiology Reference Article/Radiopaedia.org. [online] Radiopaedia.org. Available from: http://radiopaedia.org/articles/choledochal-cyst [Accessed 26 May 2015].

[21] Darge K, Anupindi S. Pancreatitis and the role of US, MRCP and ERCP. Pediatr Radiol. 2009; 39(2): 153-157. PMid:19308376 http://dx.doi.org/10.1007/s00247-009-1145-5 
[22] Costomanga G, Familiari P, Tringali A, et al. The Role of Interventional Endoscopy. In: Beart A and Laghi A. New Concept in Diagnosis and Therapeutic of Pancreatic Adenocarcinoma. 1st ed. Springer-Verlag; Berlin: 2010.p.147.165.

[23] Al-Quorain A, Ismail M, Al-Mulhim A, et al. Prospective Comparative Study of MRCP and ERCP in Biliary and Pancreatic Duct Abnormalities. Scientific Journal of King Faisal University. 2010; 11: 193-200. 